\title{
Review of: "Genomic analysis of field pennycress (Thlaspi arvense) provides insights into mechanisms of adaptation to high elevation"
}

प्र०

Potential competing interests: The author(s) declared that no potential competing interests exist.

The adaptation mechanism of organisms to extreme habitats has always been the core issue of ecological research. Phenotypic plasticity, migratory adaptation and evolutionary adaptation are the main known adaptation mechanisms. However, because the evolutionary adaptation of organisms is often an extremely long process, it is difficult to verify the evolutionary adaptation process of plants from the molecular perspective. At the same time, the mutated genes are also hidden in the huge genetic material. It is also a challenge for us to screen and determine the mutated genes that decide to adapt to evolution.

The authors selected Thalaspi arvense, a species that can be distributed at a large altitude range and the samples were collected from Qinghai-Tibet Plateau and eastern coast to carry out adaptive evolution research. This is a very wise choice, because the uplift of the Qinghai-Tibet Plateau makes most of the unadaptable plants extinction, but Thalaspi arvense can survive. In adapting to the rising altitude, the species should have adaptive evolution at the genetic level.

Through systematic experiments, the research team found that the FLC gene of the species was mutated from GT to CT at the fifth intron shear recognition site, and the amino acid translation was terminated in advance, resulting in the loss of FLC protein function. The single base mutation was fixed in the high-altitude population, resulting in early flowering of the high-altitude population, so as to adapt to the special environment of very short growth season in the Qinghai Tibet Plateau. This discovery not only better complements the molecular mechanism of plant adaptation to high altitude, but also provides screening sites for relevant research, so it has important scientific significance. 